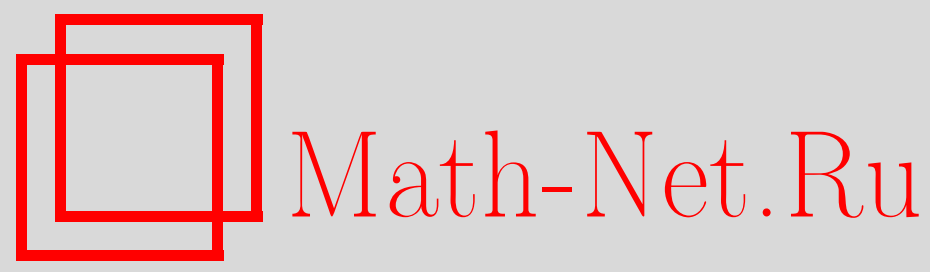

Я. Годуля, В. В. Старков, О точности некоторых оценок Кэмпбелла и Поммеренке, Матем. заметки, 1998, том 63, выпуск 5, 665-672

DOI: https://doi.org/10.4213/mzm1332

Использование Общероссийского математического портала Math-Net.Ru подразумевает, что вы прочитали и согласны с пользовательским соглашением http://www.mathnet.ru/rus/agreement

Параметры загрузки:

IP : 3.80 .253 .173

26 апреля 2023 г., $17: 50: 52$

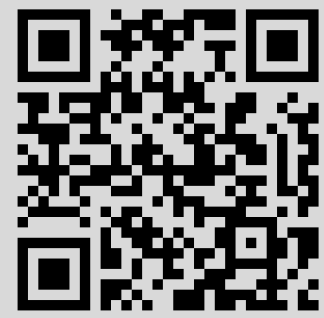




\title{
О ТОЧНОСТИ НЕКОТОРЫХ ОЦЕНОК КЭМПБЕЛЛА И ПОММЕРЕНКЕ
}

\author{
Я. Годуля, В.В. Старков
}

\begin{abstract}
Статья посвящена вопросам точности некоторых известных оценок в универсальных линейно-инвариантных семействах $\mathscr{U}_{\alpha}$ регулярных функций. В статье установлено, что полученная в 1964 г. Поммеренке оценка $\left|\arg f^{\prime}(z)\right|, z \in \Delta=\{z:|z|<1\}$, является точной; указана экстремальная функция. В $\mathcal{U}_{\alpha}$ получена нижняя оценка производной Шварца. Для $f \in \mathscr{U}_{\alpha}$ получена точная оценка порядка функции $f_{r}(z)=f(r z) / r$, $r \in(0,1)$; эта оценка используется далее при решении других задач.
\end{abstract}

Библиография: 13 названий.

Эта статья возникла в ходе нашей работы над обзором "Линейно-инвариантные семейства функций" и посвящена вопросам точности некоторых известных оценок в линейно-инвариантных семействах регулярных функций.

В [1] Ч. Поммеренке ввел понятие линейно-инвариантного семейства регулярных в круге $\Delta=\{z:|z|<1\}$ функций. Множество $\mathfrak{M}$ регулярных в $\Delta$ функций $f(z)=z+$ $\sum_{n=2}^{\infty} a_{n}(f) z^{n}$ называется линейно-инвариантны.м семейством, если для любой функции $f \in \mathfrak{M}$ вьполнены условия:

1) $f^{\prime}(z) \neq 0$ в $\Delta$ (локальная конформность);

$2)$ для любого конформного автоморфизма $\varphi(z)$ круга $\Delta$ функция

$$
\Lambda_{\varphi}[f(z)]=\frac{f(\varphi(z))-f(\varphi(0))}{f^{\prime}(\varphi(0)) \varphi^{\prime}(0)}=z+\cdots \in \mathfrak{M} .
$$

Для локально однолистной в $\Delta$ функции $f$ обозначим

$$
\mathfrak{M}[f]=\left\{\Lambda_{\varphi}[f]: \varphi-\text { любой конформньй автоморфизм } \Delta\right\}, \quad \operatorname{ord} f=\operatorname{ord} \mathfrak{M}[f] .
$$

Известно [1, с.115], что

$$
\operatorname{ord} f=\sup _{z \in \Delta}\left|-\bar{z}+\frac{1-|z|^{2}}{2} \frac{f^{\prime \prime}(z)}{f^{\prime}(z)}\right| .
$$

Порядком линейно-инвариантного семейства $\mathfrak{M}$ в $[1]$ назьвается $\sup _{f \in \mathfrak{M}}\left|a_{2}(f)\right|$. Универсальнылм линейно-инвариантным семейством $\mathscr{U}_{\alpha}$ Поммеренке называл объединение всех линейно-инвариантных семейств $\mathfrak{M}$ таких, что ord $\mathfrak{M} \leqslant \alpha, \alpha \geqslant 1$, иначе $\mathscr{U}_{\alpha}=\varnothing$. Многие известные классы конформных отображений являются линейно-инвариантньми семействами. Например, класс вьпуклых функций $K=\mathscr{U}_{1}$, класс $S$ однолистных и регулярных в $\Delta$ функций, ord $S=2$. 
1. Теорема вращения. Важнейшим из рассматриваемых в этой статье вопросов является вопрос о точности оценки в теореме вращения в $\mathscr{U}_{\alpha}$. Пусть $x, q \in[0,1)$. Обозначим

$$
\Xi(x, q)=\int_{0}^{x} \frac{\sqrt{1-q^{2} \xi^{2}}}{1-\xi^{2}} d \xi=q \arcsin x+\frac{1}{2} \sqrt{1-q^{2}} \log \frac{\sqrt{1-q^{2} x^{2}}+x \sqrt{1-q^{2}}}{\sqrt{1-q^{2} x^{2}}-x \sqrt{1-q^{2}}} .
$$

В предположении, что $\arg f^{\prime}(z)$ непрерьвен по $z \in \Delta$ и $\arg f^{\prime}(0)=0$, Поммеренке [1, c.126] в 1964 г. получил оценку $\left|\arg f^{\prime}(z)\right|$ в $\mathscr{U}_{\alpha}$.

Теорема А (вращения). Для каждой функиии $f \in \mathscr{U}_{\alpha}$ и любого $z \in \Delta$ справедливо неравенство

$$
\left|\arg f^{\prime}(z)\right| \leqslant 2 \alpha \Xi\left(|z|, \frac{1}{\alpha}\right) \leqslant \sqrt{\alpha^{2}-1} \log \frac{1+|z|}{1-|z|}+2 \arcsin |z|
$$

(последнее неравенство строгое, если $z \neq 0$ ). При этом для любого $z \in \Delta$ существует $f \in \mathscr{U}_{\alpha}$, для которой

$$
\sqrt{\alpha^{2}-1} \log \frac{1+|z|}{1-|z|} \leqslant\left|\arg f^{\prime}(z)\right| \text {. }
$$

Вопрос о точности оценки (1) оставался открытым. В 1973г. Д.М. Кэмпбелл и М.Р. Циглер в [2] исследовали свойства функции

$$
G(r, \mathfrak{M})=G(r)=\sup _{f \in \mathfrak{M}} \max _{|z|=r} \arg f^{\prime}(z),
$$

где $\mathfrak{M}$ - линейно-инвариантное семейство. Рассматривая случай $\mathfrak{M}=\mathscr{U}_{\alpha}$, они привели пример функции $f \in \mathscr{U}_{\alpha}$, для которой неравенство $(2)$ является строгим при $0<|z|<1 / \alpha$. Таким образом, они получили уточнение теоремы А для $0<|z|<1 / \alpha$ :

$$
\sqrt{\alpha^{2}-1} \log \frac{1+|z|}{1-|z|}<G\left(r, \mathscr{U}_{\alpha}\right) \leqslant 2 \alpha \Xi\left(|z|, \frac{1}{\alpha}\right) .
$$

Вопросу точности оценки $\left|\arg f^{\prime}(z)\right|$ в $\mathscr{U}_{\alpha}$, в частности, посвящена работа Кэмпбелла и Пфальцграффра [3].

Мы доказьваем точность левого неравенства в (1), указьваем экстремальную функцию.

В [4] (см. также [5]) было введено линейно-инвариантное семейство $\mathscr{U}_{\alpha}^{\prime} \subset \mathscr{U}_{\alpha}(\alpha \geqslant 1)$, ord $\mathscr{U}_{\alpha}^{\prime}=\alpha$. Имеем

$$
f \in \mathscr{U}_{\alpha}^{\prime} \Longleftrightarrow f^{\prime}(z)=\exp \left(-2 \int_{0}^{2 \pi} \log \left(1-z e^{-i t}\right) d \mu(t)\right),
$$

где $\mu(t)$ - любая комплекснозначная функция ограниченной вариации на $[0,2 \pi]$, удовлетворяющая неравенству

$$
\left|\int_{0}^{2 \pi} d \mu(t)-1\right|+\int_{0}^{2 \pi}|d \mu(t)| \leqslant \alpha .
$$


Класс $\mathscr{U}_{\alpha}^{\prime}$ компактен в топологии равномерной сходимости внутри $\Delta$, он естественным образом возникает в задаче об аппроксимации производной функции $f \in \mathscr{U}_{\alpha}$ степенями производных выпуклых функций. Можно ожидать, что многие экстремальные функции в $\mathscr{U}_{\alpha}^{\prime}$ являются экстремальными и в $\mathscr{U}_{\alpha}$. В частности, в [6] доказано, что

$$
\max _{f \in \mathcal{U}_{\alpha}^{\prime}}\left|a_{3}\right|=\frac{\alpha\left(\alpha+\sqrt{\alpha^{3}+3}\right)}{3} .
$$

Это опровергает гипотезу Кэмпбелла, Цима, Пфальцграффа [7] о том, что максимум в $(4)$ равен $\left(2 \alpha^{2}+1\right) / 3$, причем экстремальной в $(4)$ является функция

$$
h(z)=h(z, t, x)=\frac{1}{i \sqrt{\alpha^{2}-1}\left(e^{-i x}-e^{-i t}\right)}\left(\left(\frac{1+z e^{-i x}}{1+z e^{-i t}}\right)^{i \sqrt{\alpha^{2}-1}}-1\right),
$$

где $x, t$ определены в [6]. Функция вида (5) является экстремальной и в других задачах в $\mathscr{U}_{\alpha}^{\prime}$ (см., например, [8], [9]). Строго говоря, нам не удалось с помощью вариационного метода, разработанного в [4], [5], показать, что в задаче $(3)$ с $\mathfrak{M}=\mathscr{U}_{\alpha}^{\prime}$ экстремальной будет функция вида (5). Однако, применение этого вариационного метода и геометрических соображений приводит к естественному предположению, что экстремальная функция в (3) (с $\left.\mathfrak{M}=\mathscr{U}_{\alpha}^{\prime}\right)$ имеет вид (5).

Покажем, что при $\alpha>1$ и $r \in[0,1)$

$$
\max _{t, x \in \mathbb{R}} \arg h^{\prime}(r)=2 \alpha \Xi\left(r, \frac{1}{\alpha}\right) \text {. }
$$

Тем самым, будет доказана точность оценки в теореме А для всех $r \in[0,1)$. Экстремальная функция будет иметь вид (5) (экстремальные значения $t_{0}$ и $x_{0}$ укажем ниже)

$$
\arg h^{\prime}(r)=-\arg \overline{h^{\prime}(r)}=-\arg \frac{1}{\left(1+r e^{i x}\right)^{1+i \sqrt{\alpha^{2}-1}}\left(1+r e^{i t}\right)^{1-i \sqrt{\alpha^{2}-1}}}=\varphi(x)+\psi(t)
$$

где

$$
\begin{aligned}
& \varphi(x)=\sqrt{\alpha^{2}-1} \log \left|1+r e^{i x}\right|+\arg \left(1+r e^{i x}\right), \\
& \psi(t)=-\sqrt{\alpha^{2}-1} \log \left|1+r e^{i t}\right|+\arg \left(1+r e^{i t}\right) .
\end{aligned}
$$

Вычисления показывают, что

$$
\max _{x \in[0,2 \pi]} \varphi(x)=\varphi\left(x_{0}\right), \quad \text { где } \quad x_{0}=\arcsin \frac{1}{\alpha}+\arcsin \frac{r}{\alpha} .
$$

Тогда

$$
\begin{gathered}
\left|1+r e^{i x_{0}}\right|=\frac{\sqrt{\alpha^{2}-r^{2}}+r \sqrt{\alpha^{2}-1}}{\alpha} \\
\arg \left(1+r e^{i x_{0}}\right)=\arcsin \frac{r \sin x_{0}}{\sqrt{1+r^{2}+2 r \cos x_{0}}}=\arcsin \frac{r}{\alpha} .
\end{gathered}
$$


Таким образом,

$$
\varphi\left(x_{0}\right)=\sqrt{\alpha^{2}-1} \log \frac{\sqrt{\alpha^{2}-r^{2}}+r \sqrt{\alpha^{2}-1}}{\alpha}+\arcsin \frac{r}{\alpha} .
$$

Аналогично, $\max _{t \in[0,2 \pi]} \psi(t)=\psi\left(t_{0}\right)$, где $t_{0}=\pi-\arcsin (1 / \alpha)+\arcsin (r / \alpha)$. Тогда

$$
\begin{gathered}
\left|1+r e^{i t_{0}}\right|=\frac{\sqrt{\alpha^{2}-r^{2}}-r \sqrt{\alpha^{2}-1}}{\alpha}, \\
\arg \left(1+r e^{i t_{0}}\right)=\arcsin \frac{r \sin t_{0}}{\sqrt{1+r^{2}+2 r \cos t_{0}}}=\arcsin \frac{r}{\alpha}
\end{gathered}
$$

и

$$
\psi\left(t_{0}\right)=-\sqrt{\alpha^{2}-1} \log \frac{\sqrt{\alpha^{2}-r^{2}}-r \sqrt{\alpha^{2}-1}}{\alpha}+\arcsin \frac{r}{\alpha} .
$$

Следовательно,

$$
\max _{t, x \in \mathbb{R}} \arg h^{\prime}(r)=\sqrt{\alpha^{2}-1} \log \frac{\sqrt{\alpha^{2}-r^{2}}+r \sqrt{\alpha^{2}-1}}{\sqrt{\alpha^{2}-r^{2}}-r \sqrt{\alpha^{2}-1}}+2 \arcsin \frac{r}{\alpha}=2 \alpha \Xi\left(r, \frac{1}{\alpha}\right) .
$$

Точность оценки в теореме А доказана.

Поскольку $S \subset \mathscr{U}_{2}$, естественно сравнить $G(r, S)$ с $G\left(r, \mathscr{U}_{2}\right)$. В [10, с. 114] доказано, что

$$
G(r, S)= \begin{cases}4 \arcsin r & \text { для } r \in[0,1 / \sqrt{2}] \\ \pi+\log \frac{r^{2}}{1-r^{2}} & \text { для } r \in[1 / \sqrt{2}, 1) .\end{cases}
$$

Таким образом, $G(r, S) \underset{r \rightarrow 0}{\sim} G\left(r, \mathscr{U}_{2}\right)$, однако $G\left(r, \mathscr{U}_{2}\right) \underset{r \rightarrow 1-}{\sim} \sqrt{3} G(r, S)$, т.е. существенное отличие проявляется при больших $r$.

В [11] высказана гипотеза, что функция (5), дающая максимум $\left|a_{3}\right|$ в $\mathscr{U}_{\alpha}^{\prime}$, является экстремальной и в $\mathscr{U}_{\alpha}$. Естественно предположить, что $\max _{f \in \mathcal{U}_{\alpha}}\left|a_{n}\right|$ достигается для функции $f \in \mathscr{U}_{\alpha}^{\prime}$, которой в интегральном представлении соответствует кусочно постоянная функция $\mu(t)$, имеющая в точности $n-1$ разрьвов (в [6] доказано, что в задаче

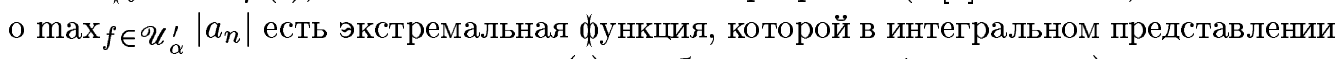
соответствует кусочно постоянная $\mu(t)$ с не более чем $n-1$ разрывами).

2. Оценка производной Шварца. Поскольку и

$$
\sup _{z \in \Delta}\left|\frac{1-|z|^{2}}{2}\right| \frac{f^{\prime \prime}(z)}{f^{\prime}(z)}|-\bar{z}|=\text { ord } f
$$

и

$$
\sup _{z \in \Delta}\left(\left(1-|z|^{2}\right)^{2}\{f(z), z\}\right)=\sigma_{f}
$$

где

$$
\{f(z), z\}=\frac{f^{\prime \prime \prime}(z)}{f^{\prime}(z)}-\frac{3}{2}\left(\frac{f^{\prime \prime}(z)}{f^{\prime}(z)}\right)^{2}
$$

- производная Шварца, являются инвариантами относительно преобразования $f \mapsto$ $\Lambda_{\varphi}[f]$, то интересно выявить соотношение между ними. Для $f \in \mathscr{U}_{\alpha}$ Поммеренке [1] получил точную оценку

$$
\alpha \leqslant \sqrt{1+\frac{\sigma_{f}}{2}} .
$$

Обозначим $\sigma=\sigma(\alpha)=\sup _{f \in \mathscr{U}_{\alpha}} \sigma_{f}$. Интересно, что неравенство между $\alpha$ и $\sigma$ незначительно трансформируется по сравнению с (6). 
ПРЕДЛОЖЕНИЕ. $\alpha \leqslant \sqrt{\sigma(\alpha) / 2}$.

ДокАЗАТЕЛЬСТВо. Если $f \in \mathscr{U}_{\alpha}, \zeta=\varphi(z)-$ конформный автоморфизм $\Delta$ и $g(z)=$ $\Lambda_{\varphi}[f(z)]$, то

$$
\left(1-|\zeta|^{2}\right)^{2}|\{f(\zeta), \zeta\}|=\left(1-|z|^{2}\right)^{2}|\{g(z), z\}| \leqslant \sigma .
$$

Отсюда при $z=0$ получаем

$$
\left|\frac{g^{\prime \prime \prime}(0)}{g^{\prime}(0)}-\frac{3}{2}\left(\frac{g^{\prime \prime}(0)}{g^{\prime}(0)}\right)^{2}\right| \leqslant \sigma \Longleftrightarrow 6\left|a_{3}-a_{2}^{2}\right| \leqslant \sigma
$$

где $g(z)=z+a_{2} z^{2}+a_{3} z^{3}+\cdots \in \mathscr{U}_{\alpha} . \mathrm{B}[8]$ найден $\max _{g \in \mathscr{U}_{\alpha}^{\prime}}\left|a_{3}-\lambda a_{2}^{2}\right|$ для $\lambda \in \mathbb{R} . \mathrm{B}$ частности,

$$
\max _{g \in \mathscr{U}_{\alpha}^{\prime}}\left|a_{3}-a_{2}^{2}\right|=\frac{\alpha^{2}}{3} .
$$

Экстремальную функцию в этой задаче подставим в $(7)$, получим $2 \alpha^{2} \leqslant \sigma$.

Это доказывает наше предложение.

3. Оценка ord $f_{r}$ и $\mathscr{U}_{\beta}$-радиуса. Для $r \in(0,1]$ преобразование $f(z) \mapsto f(r z) / r=$ $f_{r}(z)$ назьвается преобразованием сжсатия. В некоторых задачах в $\mathscr{U}_{\alpha}$ важно знать, как зависит ord $f_{r}$ от $r$ и $\alpha=\operatorname{ord} f$.

ТЕорема Б [12]. Если ord $f=\alpha$, mо ord $f_{r}$ - непрерывно возрастающая функиия на $(0,1]$. Более того, ord $f_{r}$ строго возрастает при $r$, больиих радиуса выпуклости функиии $f$ и

$$
\text { ord } f_{r} \leqslant(\alpha-1) r+1 \text {. }
$$

Отсюда, в частности, следует инвариантность $\mathscr{U}_{\alpha}$ относительно преобразования сжатия.

Мы улучшаем неравенство (8), получая точную оценку

$$
\left\{\begin{array}{l}
\operatorname{ord} f_{r}=1 \text { при } r \in\left(0, \alpha-\sqrt{\alpha^{2}-1}\right], \\
\frac{1}{2}\left(\tau+\frac{1}{\tau}\right) \text { при } r \in\left[\alpha-\sqrt{\alpha^{2}-1}, 1\right]
\end{array}\right.
$$

где $\tau=r\left(\alpha+\sqrt{\alpha^{2}-1}\right)$.

ДОКАЗАТЕЛЬСТВо НЕРАВЕНСТВА (9). В $[1$, с. 133$]$ доказано, что радиус вьпуклости $\mathscr{U}_{\alpha}$ равен $\alpha-\sqrt{\alpha^{2}-1}$. Поэтому при $r \in\left(0, \alpha-\sqrt{\alpha^{2}-1}\right]$ функции $f_{r}(z) \in K=\mathscr{U}_{1}$. Следовательно, при этих $r$ ord $f_{r}=1$; это доказьвает первую часть (9). Пусть теперь $r \in\left(\alpha-\sqrt{\alpha^{2}-1}, 1\right]$. Имеем

$$
\begin{aligned}
\operatorname{ord} f_{r} & =\sup _{z \in \Delta}\left|-\bar{z}+\frac{1-|z|^{2}}{2} \frac{f^{\prime \prime}(r z)}{f^{\prime}(r z)} r\right| \\
& =\sup _{z \in \Delta}\left|r \frac{1-|z|^{2}}{1-r^{2}|z|^{2}}\left(-r \bar{z}+\frac{1-r^{2}|z|^{2}}{2} \frac{f^{\prime \prime}(r z)}{f^{\prime}(r z)}\right)-\bar{z}\left(1-r^{2} \frac{1-|z|^{2}}{1-r^{2}|z|^{2}}\right)\right| \\
& \leqslant \sup _{x \in[0,1)} V(x), \quad \text { где } V(x)=\frac{\alpha r\left(1-x^{2}\right)+x\left(1-r^{2}\right)}{1-r^{2} x^{2}} .
\end{aligned}
$$


Так как

$$
V^{\prime}(x)=\left(1-r^{2}\right) \frac{1-2 \alpha r x+r^{2} x^{2}}{\left(1-r^{2} x^{2}\right)^{2}}
$$

TO

$$
\sup _{x \in[0,1)} V(x)=V\left(\frac{\alpha-\sqrt{\alpha^{2}-1}}{2}\right)=\frac{1}{2}\left(r\left(\alpha+\sqrt{\alpha^{2}-1}\right)+\frac{1}{r\left(\alpha+\sqrt{\alpha^{2}-1}\right)}\right) .
$$

В заключении доказательства заметим, что равенство в (9) достигается для функции

$$
f_{0}(z)=\frac{1}{2 \alpha}\left(\left(\frac{1+z}{1-z}\right)^{\alpha}-1\right)
$$

По аналогии с радиусами однолистности и вьпуклости Кэмпбелл [12] ввел понятие $\mathscr{U}_{\beta}$-радиуса $\mathscr{R}(\beta)$ компактного семейства $\mathfrak{M}$ :

$$
\mathscr{R}(\beta)=\max \left\{\rho: \frac{f(\rho z)}{\rho} \in \mathscr{U}_{\beta} \quad \forall f \in \mathfrak{M}\right\} .
$$

Если $\beta \geqslant \alpha$, то $\mathscr{U}_{\beta} \supset \mathscr{U}_{\alpha}$. Поэтому $\mathscr{U}_{\beta}$-радиус семейства $\mathscr{U}_{\alpha}$ равен 1, и интерес представляет только случай $\beta<\alpha$. $\mathscr{U}_{1}$-радиус семейства $\mathscr{U}_{\alpha}$ является радиусом вьпуклости $\mathscr{U}_{\alpha}$ (см. [1, с. 133]). В [12] доказано, что при $\beta \leqslant \alpha \quad \mathscr{U}_{\beta}$-радиус семейства $\mathscr{U}_{\alpha}$ удовлетворяет неравенствам

$$
\max \left\{\alpha-\sqrt{\alpha^{2}-1}, \frac{\beta-1}{\alpha-1}\right\} \leqslant \mathscr{R}(\beta) \leqslant\left(\beta+\sqrt{\beta^{2}-1}\right)\left(\alpha-\sqrt{\alpha^{2}-1}\right) .
$$

Мы уточняем этот результат с помощью неравенства (9).

ТеоремА. При $1 \leqslant \beta \leqslant \alpha \quad \mathscr{U}_{\beta}$-радиус семейства $\mathscr{U}_{\alpha}$ равен

$$
\mathscr{R}(\beta)=\left(\beta+\sqrt{\beta^{2}-1}\right)\left(\alpha-\sqrt{\alpha^{2}-1}\right) .
$$

ДОКАЗАТЕЛЬСТВО. Если $1 \leqslant \beta \leqslant \alpha$, то $\max _{f \in \mathscr{U}_{\alpha}}$ ord $f_{\mathscr{R}(\beta)}=\beta$. Из точности неравенства (9) следует также, что $\max _{f \in \mathcal{U}_{\alpha}}$ ord $f_{\mathscr{R}(\beta)}=\frac{1}{2}(\tau+1 / \tau)$, где

$$
\tau=\mathscr{R}(\beta)\left(\alpha+\sqrt{\alpha^{2}-1}\right) \geqslant \mathscr{R}_{1}\left(\alpha+\sqrt{\alpha^{2}-1}\right)=1 .
$$

Поэтому $\mathscr{R}(\beta)$ удовлетворяет уравнению $\beta=\frac{1}{2}(\tau+1 / \tau), \tau \geqslant 1$. Отсюда находим $\tau=\beta+\sqrt{\beta^{2}-1}$. Следовательно, $\mathscr{R}(\beta)=\left(\beta+\sqrt{\beta^{2}-1}\right)\left(\alpha-\sqrt{\alpha^{2}-1}\right)$.

Теорема доказана.

Обозначим $r_{u}(\alpha)$ радиус однолистности $\mathscr{U}_{\alpha}$, т.е.

$$
r_{u}(\alpha)=\max \left\{\rho: \frac{f(\rho z)}{\rho} \in S \quad \forall f \in \mathscr{U}_{\alpha}\right\} .
$$

Если $\zeta_{0}-$ решение уравнения $\Xi\left(\zeta_{0}, 1 / \alpha\right)=\pi / \alpha, \zeta_{1}=\zeta_{0} /\left(1+\sqrt{1-\zeta_{0}^{2}}\right)$, то (см. [1, с. 135])

$$
\zeta_{1} \leqslant r_{u}(\alpha) \leqslant \operatorname{th} \frac{\pi}{2 \sqrt{\alpha^{2}-1}}, \quad r_{u}(\alpha)=\frac{\pi}{2 \alpha}+O\left(\frac{1}{\alpha^{2}}\right) \text { при } \alpha \rightarrow \infty .
$$

Из доказанной теоремы получается качественньй результат о $r_{u}(\alpha)$. 
СлЕДСТВИЕ. Обозначим $\rho(\alpha)=\left(\alpha+\sqrt{\alpha^{2}-1}\right) r_{u}(\alpha)$. Функиия $\rho(\alpha)$ возрастает nо $\alpha \in[1, \infty), \rho(1)=1, \lim _{\alpha \rightarrow \infty} \rho(\alpha)=\pi$.

Действительно, если $1 \leqslant \beta \leqslant \alpha$ и $f \in \mathscr{U}_{\alpha}$, то

$$
\frac{f\left(\mathscr{R}(\beta) r_{u}(\beta) z\right)}{\mathscr{R}(\beta) r_{u}(\beta)} \in S
$$

Следовательно, $\mathscr{R}(\beta) r_{u}(\beta) \leqslant r_{u}(\alpha)$. Поэтому $\left(\beta+\sqrt{\beta^{2}-1}\right) r_{u}(\beta) \leqslant\left(\alpha+\sqrt{\alpha^{2}-1}\right) r_{u}(\alpha)$. При $\alpha=1 r_{u}(1)=1$ и $\rho(1)=1$. Поскольку $S \subset \mathscr{U}_{2}$, имеем

$$
\mathscr{R}_{2}=\frac{2+\sqrt{3}}{\alpha+\sqrt{\alpha^{2}-1}} \geqslant r_{u}(\alpha)
$$

Следовательно, функция $\rho(\alpha)$ ограничена и имеет конечный предел $c=\lim _{\alpha \rightarrow \infty} \rho(\alpha)$. Тогда при $\alpha \rightarrow \infty$

$$
r_{u}(\alpha)=\frac{c+o(1)}{\alpha+\sqrt{\alpha^{2}-1}}=\frac{c}{2 \alpha}+o\left(\frac{1}{\alpha}\right) .
$$

Сравнивая это равенство с $(10)$, находим $c=\pi$. Заметим, что функция $\rho(\alpha)$ останется возрастающей, если в ее определении заменить $r_{u}(\alpha)$, например, на радиус звездообразности $r_{*}(\alpha)$. Но в этом случае предел $c_{*} \geqslant 2$, поскольку $r_{*}(\alpha) \geqslant \frac{1}{\alpha}$ (cм. [1, с. 134]). Можно показать, что $c_{*}>2.04$.

4. Локально однолистные функции с локально однолистными производными. Пусть $\mathfrak{C}$ - некоторый класс конформных в $\Delta$ отображений $f(z)=z+a_{2} z^{2}+\cdots$.

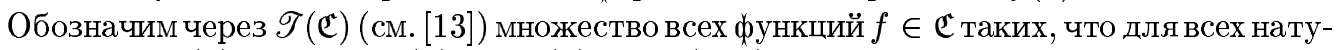
ральных $n f^{(n)}(z) \neq 0$ и $\left(f^{(n)}(z)-f^{(n)}(0)\right) / f^{(n+1)}(0) \in \mathfrak{C}, A(\mathfrak{C})=\sup \left\{\left|a_{2}\right|: f \in \mathscr{T}(\mathfrak{C})\right\}$. В [13] доказано, что $\pi / 2 \leqslant A(S)<1.7208,1 / 2 \leqslant A\left(\mathscr{U}_{1}\right)<0.68379$. В [12] получено неравенство

$$
\frac{\alpha+\sqrt{\alpha^{2}-1}}{2} \leqslant A\left(\mathcal{U}_{\alpha}\right)=A_{\alpha} \leqslant \alpha .
$$

Мы покажем, что в правой части (11) неравенство строгое для всех $\alpha \geqslant 1$. Действительно, в противном случае сушествует функция $f(z)=z+\sum_{n=2}^{\infty} a_{n} z^{n} \in \mathscr{T}\left(\mathscr{U}_{\alpha}\right)$, для которой $a_{2}=A(\alpha)=\alpha$, так как $\mathscr{T}\left(\mathscr{U}_{\alpha}\right)$ компактен в топологии равномерной сходимости внутри $\Delta$. Поэтому

$$
\alpha=\frac{1}{2} f^{\prime \prime}(0) \leqslant \sup _{z \in \Delta}\left|-\bar{z}+\frac{1-|z|^{2}}{2} \frac{f^{\prime \prime}(0)}{f^{\prime}(0)}\right| \leqslant \alpha .
$$

Следовательно,

$$
\left.\frac{d}{d r} \operatorname{Re}\left(-r+\frac{1-r^{2}}{2} \frac{f^{\prime \prime}(r)}{f^{\prime}(r)}\right)\right|_{r=0}=0 \Longleftrightarrow \operatorname{Re} f^{\prime \prime \prime}(0)=2+\left(f^{\prime \prime}(0)\right)^{2}
$$

и

$$
\operatorname{Re} a_{3}=\frac{1}{3}+\frac{2}{3} a_{2}^{2}
$$


С другой стороны,

$$
\operatorname{ord} \frac{f^{(n)}(z)-f^{(n)}(0)}{f^{(n+1)}(0)} \leqslant \alpha,
$$

т.e.

$$
\sup _{z \in \Delta}\left|-\bar{z}+\frac{1-|z|^{2}}{2} \frac{f^{(n+2)}(z)}{f^{(n+1)}(z)}\right| \leqslant \alpha=a_{2} .
$$

В частности, при $n=1$ отсюда следует, что $\left|f^{\prime \prime \prime}(0)\right| \leqslant 2 \alpha f^{\prime \prime}(0)$, и $\left|a_{3}\right| \leqslant \frac{2}{3} a_{2}^{2}$, что противоречит (12).

\section{СПИСОК ЦИТИРОВАННОЙ ЛИТЕРАТУРЫ}

[1] Pommerenke Ch. Linear-invariante Familien analytischer Funktionen. I // Math. Ann. 1964. V. 155. P. 108-154.

[2] Campbell D. M., Ziegler M. R. The argument of derivative linear-invariant families of finite order and the radius of close-to-convexity // Ann. Univ. Mariae Curie-Skłodowska. Sect. A. 1976. V. 28. P. 5-22.

[3] Campbell D. M., Pfaltzgraff J. A. Boundary behaviour and linear invariant families // J. Anal. Math. 1976. V. 29. P. 67-92.

[4] Старков В. В. О некоторых подклассах линейно-инвариантных семейств, имеющих интегральное представление // Деп. ВИНИТИ № 3341-81. М.: ВИНИТИ 1981.

[5] Старков В. В. О некоторых линейно-инвариантных семействах функций, имеющих интегральное представление // Изв. вузов. Матем. 1983. № 5. С. 82-85.

[6] Старков В.В. К оценке коэффициентов в классе $U_{\alpha}^{\prime}$ локально однолистных функций // Вестн. ЛГУ. Матем., мех., астрон. 1984. №13. С. 48-54.

[7] Campbell D. M., Cima J. A., Pfaltzgraff J. A. Linear space and linear-invariant families of locally univalent analytic functions // Manuscripta Math. 1971. V. 4. P. 1-30.

[8] Старков В.В. Об одном неравенстве для коэффициентов функций некоторого линейно-инвариантного семейства // C. R. Acad. Bulgare Sci. 1984. V. 37. № 8. P. 999-1002.

[9] Godula J., Starkov V. V. On the Jakubowski's functional in a linearly invariant family // Zeszyty Nauk. Politech. Rzeszowskiej. Mat. Fiz. 1990. V. 73. P. 19-27.

[10] Голузин Г. М. Геометрическая теория функций комплексного переменного. М.: Наука 1966.

[11] Старков В. В. К проблемекоэффициентов в $U_{\alpha}^{\prime}$ // Деп. ВИНИТИ№ 972-82. М.: ВИНИТИ, 1981.

[12] Campbell D. M. Locally univalent functions with locally univalent derivatives // Trans. Amer. Math. Soc. 1971. V. 162. P. 395-409.

[13] Shah S. M., Trimble S. Y. Univalent functions with univalent derivatives. III // J. Appl. Math. Mech. 1969/1970. V. 19. P. 451-460.

Институт математики университета М. Кюри-Склодовской, г. Люблин

Поступило

Петрозаводский государственный университет

28.10 .96

E-mail: godula@golem.umcs.lublin.pl, starkov@mainpgu.karelia.ru 